\title{
DESIGN, DEVELOPMENT AND OPERATIONAL EXPERIENCES OF THE POWER CONVERTERS USED ON THE SRS *
}

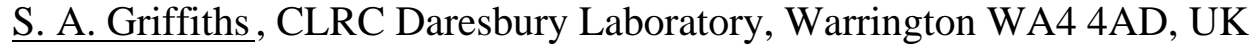

\begin{abstract}
This report provides an overview of the past and present developments in power converter technology used on the CLRC Daresbury Laboratory Synchrotron Radiation Source (SRS). These converters have ratings which range from hundreds of watts to $750 \mathrm{~kW}$ and supply both conventional inductive loads and super-conducting magnets. The paper summaries the impact of advances in semi-conductor component specification on the design and operation of these power converters and indicates the recent developments which have occurred.
\end{abstract}

\section{SUPER CONDUCTING MAGNET POWER CONVERTERS}

There are two super conducting Wiggler magnets in the SRS, which are supplied by commercially available switch-mode power converters. These supplies have a rated long-term stability of 1 part in $10^{3}$ which is insufficient for the duty required. A combined external current and voltage stabiliser is incorporated using a DCCT to measure the load current and potential divider to feedback the voltage. The switch-mode units serve only as a high power amplifier and long term stability of 1 part in $10^{4}$ is easily achieved.

\subsection{Energy Storage}

The dissipation of the stored energy of the smaller 5 Tesla magnet is provided by freewheel diodes connected to the four individual main coils of the magnet; full discharge is achieved in approximately 7 minutes.

The second magnet rated at 6 Tesla consists of a main and auxiliary winding shown in Fig. 1. It is not possible to power the coils separately as the connection from the interlink is only rated to conduct $10 \%$ of the nominal current. A freewheel diode on the main magnet would provide discharge in approximately 30 minutes and prolong refill times.

To improve the discharge rate, high power MOSFETs are used to control the operation of a dumping circuit. The design utilises the main current feedback system to adjust the switching time of the MOSFETs and track the reference as it ramps to zero in 6 minutes.

To avoid a possible quench, the discharge rate must be controlled particularly at maximum field. If an interlock trip should occur the supply must remains 'on' until zero current.
The auxiliary winding is connected with anti parallel schottky diodes to provide a path for the discharging main current. This limits the voltage, which is generated across the auxiliary coil because of the current difference in the main and auxiliary windings. The low forward voltage drop of the schottky rectifiers is insufficient to meet the need for high impedance during the ramp. As a compromise the trim power supply is overrated and the DCCT for the auxiliary current is positioned close to the magnet terminals to prevent inaccuracies resulting from diode conduction.

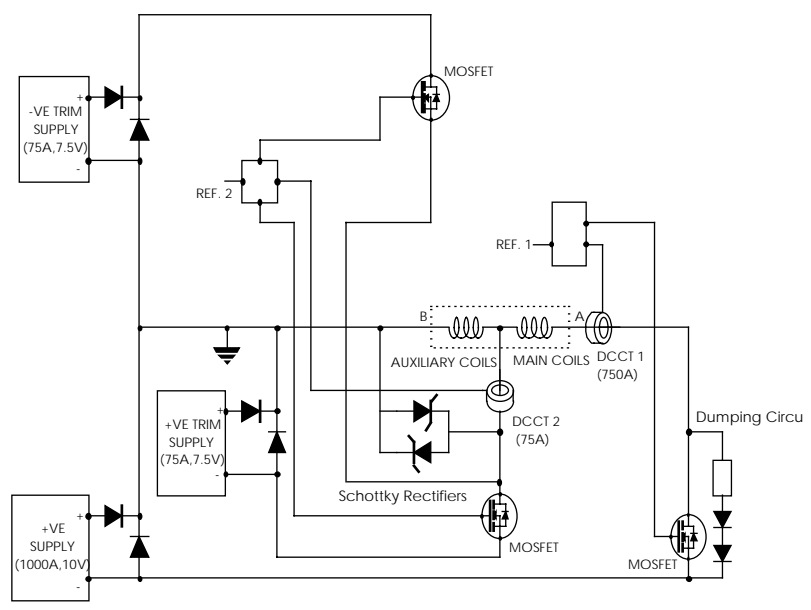

Fig.1 Schematic diagram of the 6 Tesla Superconducting Wiggler Power Converter

\section{QUADRUPOLE SHUNTS}

A significant vertical tune shift can arise from the focussing effects of the insertion devices. (wigglers and undulators) This can be compensated by local correction on a quadrupole adjacent to the wiggler. The main magnet families of the storage ring are series connected and to adjust current to individual quadrupoles a controllable active current shunt is required.

\subsection{Design Changes}

The initial shunt design was a transistor bank containing 32 parallel bipolar transistors. Their base drive was generated by a transformer-coupled chopper and rectifier to amplify the current and provide electrical isolation.

This system has since been simplified and the new design is shown in Fig.2. It consists of two parallel high 
power MOSFETs with an isolation amplifier providing the interface to the control circuit. The high input impedance and large power gain of the MOSFET is ideally suited to this type of application.

The main contactor is used for electrical isolation as part of the personnel safety system. The on/off switching is achieved by clamping the base drive.

The capacitor and zener diodes on the magnet side of the contactor ease the breaking of the dc arc in the contactor and prevent high voltages being generated.

\subsection{Control Electronics}

The control circuitry for the quadrupole shunts was designed multi-functional and provides a site standard for all power converter applications. Its features include a ramp generator, voltage feedback conditioning and isolated analogue output drive. The system also houses a QtecQ Macc plus DCCT burden card, providing a simple and compact feedback interface. The electronics are housed in a Euro-crate with a PCB backplane providing an interface to external control electronics. This makes the control crate portable and easy to replace.

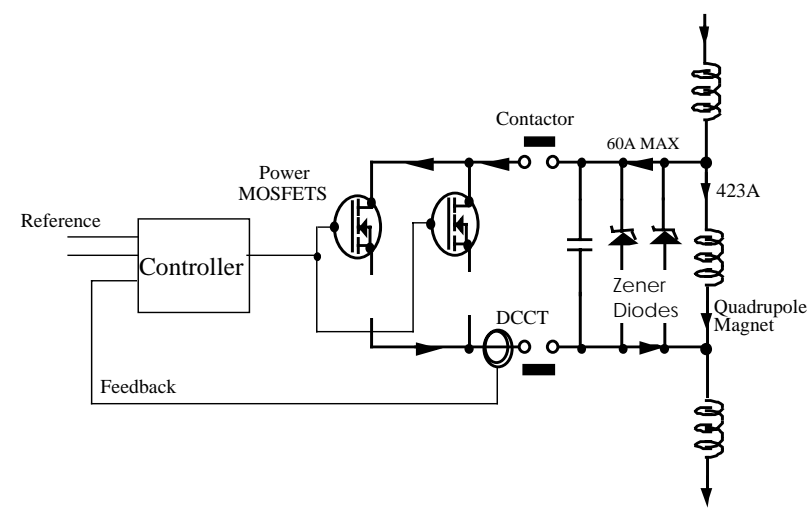

Fig.2 Schematic Diagram of Quadrupole Shunt Circuit

\section{MAIN STORAGE RING POWER CONVERTERS}

The main magnets of the storage ring were initially powered by roller regulator, transistor trimmer type power converters. This type of supply is expensive to maintain and has poor efficiency compared to modern design. Their reliability had become unacceptable due to age for an accelerator expected to deliver 6000 scheduled beam hours per year. It was decided to replace these supplies with a $50 \mathrm{~Hz}$ line commutated thyristor technology, designed to the CERN, LEP specifications [1,2,3].

There are five converters in this power range, two at $750 \mathrm{~kW}$, one at $250 \mathrm{~kW}$ and two at $90 \mathrm{~kW}$. These power the SRS storage ring dipoles, quadrupoles and sextupoles.

\subsection{Design Benefits}

The improved performance of the new converters reduced the ramp time from 14 minutes, with the old regulator type design, to just 70 seconds. The hysteresis cycling before each refill is also faster, now being 30 seconds instead of 4 minutes.

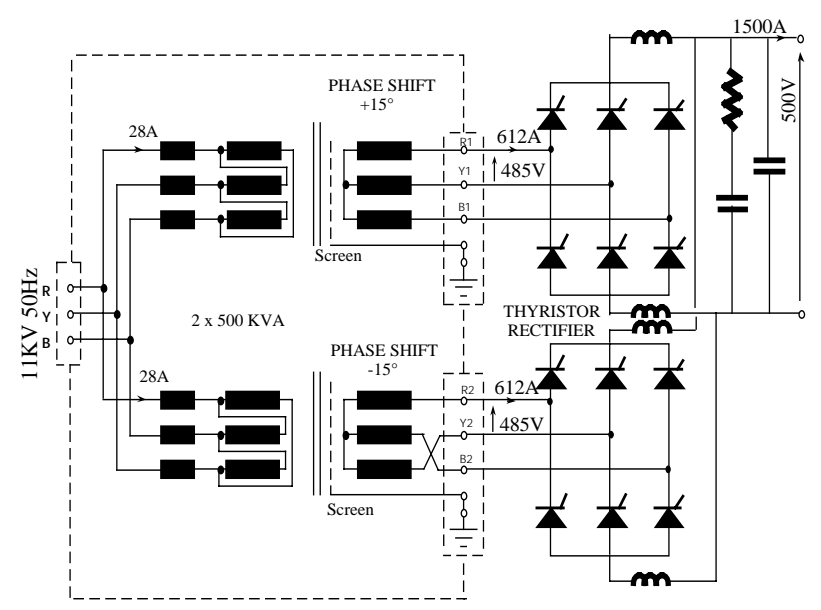

Fig.3 Electrical Circuit of 750kW Power Converter

All of the new converters are naturally air cooled, thus making it possible to dispense completely with the closed circuit de-mineralised water cooling system used by the old equipment. This, together with the high efficiency of the converter, yields a reduction in power of about $30 \mathrm{~kW}$.

The inclusion of two DCCTs, one for control and the other for monitoring, allows direct on-line calibration checks at the nominal output current. This can then be used to identify drifts in the DAC, ADC and loop parameters. The extensive range of command protocol and monitoring facilities provides a comprehensive diagnostic system for troubleshooting.

Since the installation of the new converters, downtime due to failures has reduced by a factor of 10 on the old design.

\section{BEAM STEERER POWER CONVERTERS}

The present system consists of two 24 volt switch-mode power converters providing a dual dc distribution network to support 224 steering modules as shown in Fig.4. The load regulation is generated by individual current control of the transistorised modules with a stability of 1 part in $10^{4}$. The DC supply from the switch-mode units is over specified for this application, but due to its complex switch-mode design and 10 years operating period the reliability is poor. 


\subsection{Proposed Replacement}

It is now proposed to replace the switch-mode supplies with a transformer rectifier set; the design simplicity and generously rated components would guarantee a high mean time between failures and provide a low cost solution. Before the power supplies are replaced the modules must be assessed for bandwidth and attenuation of the $300 \mathrm{~Hz}$ ripple produced from the three-phase rectifier.

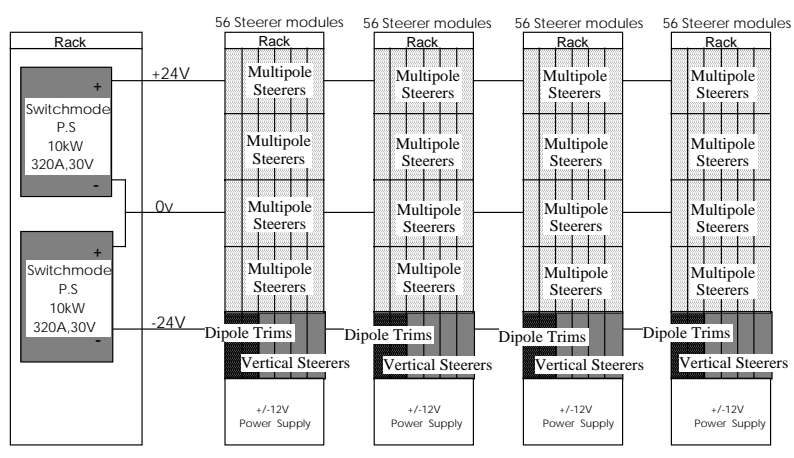

Fig.4 Electrical Distribution of the Steerer Magnet Power Converters.

The major problem with this type of dc distribution systems is the large amount of stored energy, which can result from the combined magnet loads. When redesigning the system all possible fault conditions must be assessed; for example:

- module fuse failure, each unit must be capable of discharging the magnet energy, preventing further damage to module.

- power converter failure, this would not cause damage as the control circuit would remain operational and the energy discharged normally.

- A loss of control reference to the modules, this can result in the stored energy being discharged into the output capacitors of the power converter causing an over voltage; the supply must have built in over voltage protection to prevents damage.

\section{FUTURE DEVELOPMENTS}

\subsection{Booster Power Converter}

The ac power converter for the $10 \mathrm{~Hz}$ Booster White circuit used on the SRS has been operational for 20 years. The design consists of a thyristor rectifier, which controls the dc voltage to the $10 \mathrm{~Hz}$ thyristor inverter circuit. The inverter then supplies the losses to the white circuit using an auxiliary winding on the energy storage choke. The ideal solution to improve the power converter would be to specify and procure an entire new unit. This is not possible and it is now proposed to upgrade the control electronics, providing a low cost alternative.

\subsection{Crowbarless Klystron Power Converter}

The power converter for the existing storage ring RF klystron is a 6-pulse transformer rectifier, with roller regulator output voltage control. Advances in power electronics components now make possible the production of switch-mode power converters exceeding $100 \mathrm{~kW}$. The use of high frequency inverters means that the stored energy at the high voltage end is greatly reduced compared to a line commutated rectifier. This makes it possible to eliminate fast protection by electronic crowbar. This is an expensive device, which requires high quality design and manufacture. A liaison with an industrial company, to jointly produce such a converter, is currently being explored.

\section{REFERENCES}

[1] D.E.Poole, S.A.Griffiths and M Heron, "New Magnet Power Converter for the SRS at Daresbury", Proceedings of European Particle Accelerator Conference, 1994, vol. 3, pp 2333-2334.

[2] H.W.Isch, J.G.Pett and P.Proundlock, "An Overview of the LEP Power Converter System", Proceedings of the IEEE Particle Accelerator Conference, Washington, 1987, vol.3, pp. 1399-1401.

[3] H.W.Isch, "Realisation of LEP Power Converters", $1^{\text {st }}$ European Particle Accelerator Conference, Rome, 1988, vol.2, pp 1166-1186. 